\title{
Inclusion Exclusion Criterion Original Result
}

National Cancer Institute

\section{Source}

National Cancer Institute. Inclusion Exclusion Criterion Original Result. NCI Thesaurus.

Code C83297.

The first outcome of the inclusion exclusion criterion. 